\title{
Wie Sachsen geteilt wurde Die „sächsische Frage" auf dem Wiener Kongress 1815
}

\author{
Matthias Donath
}

Vgl. die Kongressakten in Johann Friedrich Klüber, Acten des Wiener Congresses. Bd. 1-8, Erlangen 1815-1819; Comte d'Angeberg [Leonard Borejko Chodźko], Le Congrès de Vienne et les traités de 1815 . Bd. 1/2, Paris 1863/64; Klaus Müller (Hrsg.), Quellen zur Geschichte des Wiener Kongresses 1814/15, Darmstadt 1986.

2 Vgl. Karlheinz Blaschke, Bewahrte Einheit. Die Oberlausitz in den 130 Jahren erzwungener Teilung 1815-1945, in: Karlheinz Blaschke, Beiträge zur Geschichte der Oberlausitz. Gesammelte Aufsätze, Görlitz/ Zittau 2000, S. 165

3 Rudolf Jenak, Ursachen und Hintergründe der sächsischen Territorial-Abtretungen an das Königreich Westphalen im Jahre 1808, in: Neues Archiv für sächsische Geschichte 74/75 (2003/2004), S. 443-453.
Vor 200 Jahren wurde auf dem Wiener Kongress die Teilung Sachsens beschlossen. König Friedrich August I. von Sachsen (1750-1827) musste 58 Prozent seines Landes abtreten. 42 Prozent der Einwohner Sachsens kamen, ohne dass sie gefragt wurden, unter preußische Herrschaft. Abgetreten wurden nahezu zwei Drittel der Oberlausitz, die gesamte Niederlausitz, der Wittenberger Kreis (Kurkreis), Teile des Meißner und des Leipziger Kreises, die Stifter Merseburg und Naumburg, der Thüringer Kreis, der Neustädter Kreis, der sächsische Anteil der Grafschaft Henneberg sowie mehrere Orte des Vogtländischen Kreises.

Durch neue Forschungen war es möglich, die Akten des Wiener Kongresses genauer auszuwerten und den Verlauf der Verhandlungen nachzuzeichnen. ${ }^{1}$ Dabei wurde deutlich, dass zahlreiche weit verbreitete Urteile überdacht werden müssen. So wird die 1815 beschlossene „blutige Zerreißung“ Sachsens oftmals allein der preußischen Seite zugeschrieben, und der Grenzverlauf diene allein dem militärischen Interesse des Königreichs Preußen, eine „strategische Aufmarschbasis für den nächsten Krieg gegen Österreich zu schaffen. ${ }^{2}$ Aus den Kongressakten geht jedoch hervor, dass die Grenzlinie mitten durch Sachsen keineswegs von Preußen gefordert worden war. Vielmehr wollte der preußische König Sachsen ungeteilt der preußischen Monarchie angliedern. Österreich, Frankreich und England konnten das durch diplomatisches Geschick verhindern und einen Kernbestand des Königreichs Sachsen erhalten. Der Grenzverlauf beruhte auf Vorschlägen Österreichs, weil diese andere deutsche Großmacht eigene militärische Ziele verfolgte.

Kurfürst Friedrich August III. von Sachsen hatte im Friedensvertrag von Posen vom 12. Dezember 1806, der ihm den Königstitel bescherte, vom französischen Kaiser Napoleon Bonaparte (1769-1821) die Zusage erhalten, den Cottbusser Kreis in sein Königreich eingliedern zu können. Dieses vollständig von sächsischem Gebiet umgebene, jedoch unter preußischer Hoheit stehende Territorium wurde am 12. September 1807 dem Königreich Sachsen unterstellt, nachdem Napoleon seinen preußischen Widersacher, König Friedrich Wilhelm III. (1770-1840), militärisch besiegt hatte. Zudem wurde dem sächsischen König die Würde eines Großherzogs von Warschau übertragen. Das neu geschaffene Großherzogtum Warschau war ein selbständiger Staat, der lediglich in Personalunion mit Sachsen verbunden war. 1808 musste der König von Sachsen kleinere Landesteile an das von Napoleon neu geschaffene Königreich Westphalen abtreten: das Amt Gommern des Kurkreises, die Grafschaft Barby, den sächsischen Anteil der Grafschaft Mansfeld sowie den sächsischen Anteil der Ganerbschaft Treffurt und der Vogtei Dorla. ${ }^{3}$ Nach der Niederlage Napoleons in der Völkerschlacht bei Leipzig und der Gefangennahme des sächsischen Königs wurde Sachsen als besiegtes Land behandelt und besetzt. Der Cottbusser Kreis fiel unverzüglich an Preußen zurück. Das von den Alliierten geschaffene und zunächst unter die Aufsicht des russischen Fürsten Nikolai Repnin-Wolkonski (1778-1845) gestellte „General-Gouvernement der Hohen Verbündeten Mächte“ bediente sich überwiegend sächsischer Beamter, die jedoch dem russischen Zaren zur Treue verpflichtet waren. Wichtige Positionen waren mit Verwaltungsfachleuten besetzt, die als „Franzosenfeinde“ galten und eine Angliederung Sachsens an Preußen befürworteten. Die Verwaltung zielte auf die Eingliederung Sachsens in die preußischen Monarchie ab. Das entsprach ganz den Interessen Russlands und Preußens, die sich in 
der Konvention von Kalisch im Februar 1813 bereits auf eine ungefähre Aufteilung der von Napoleon befreiten Länder geeinigt hatten. Zar Alexander I. von Russland (1777-1825) hatte die Zusage erhalten, dass er den größten Teil des Großherzogtums Warschau erhalten werde. Er wollte ein formal eigenständiges Königreich Polen schaffen und dieses in Personalunion mit Russland verbinden. Da Preußen bei dieser Aufteilung Gebiete verlor, die es bei der zweiten und dritten Teilung Polens erhalten hatte, war es naheliegend, Preußen mit Sachsen zu entschädigen.

Als sich die verbündeten Mächte im September 1814 in Wien versammelten, um auf Einladung des Kaisers Franz I. von Österreich (1768-1835) und des österreichischen Außenministers Clemens Wenceslaus Lothar Fürst von Metternich (1779-1859) einen umfassenden Friedensvertrag auszuhandeln und eine Neuordnung der Ländergrenzen in Europa vorzunehmen, glaubte die preußische Seite, ihre Forderung nach der Einverleibung Sachsen rasch durchsetzen zu können. Der Vertreter Englands, Robert Viscount Castlereagh (1769-1822), stimmte am 11. Oktober 1814 in einer Note der Annexion Sachsens zu. ${ }^{4}$ Auch von Österreich erhoffte man sich eine Zustimmung. Doch in einer Note an den Bevollmächtigten des preußischen Königs, den Fürsten Carl August von Hardenberg (1750-1822), vom 22. Oktober 1814 formulierte Metternich die Bedingungen für die Zustimmung zu einer Friedensordnung. ${ }^{5}$ Wie es in einem Nebensatz heißt, gebe man zu bedenken, ob nicht ein „Kern“ des Königreiches Sachsen erhalten bleiben könne. Dabei bliebe den Mächten die Aufgabe erspart, den König von Sachsen andernorts zu entschädigen. Freilich überlas Hardenberg diesen Nebensatz und erblickte in der Note die Zustimmung Österreichs zu einer Angliederung Sachsens.

In einem nicht datierten Memorandum vom Oktober 1814 trug der österreichische Diplomat Johann Freiherr von Wessenberg (17731858) die Argumente zusammen, die gegen eine Annexion Sachsens sprachen. In diesem machte er erstmals den Vorschlag für eine Landesteilung. ${ }^{6}$ Dem König von Preußen sollten die Niederlausitz, der Wittenberger Kreis (bis 1806 Kurkreis), die Ämter Barby, Gommern, Querfurt und Jüterbog, der sächsische Anteil der Grafschaft Mansfeld sowie sechs Ämter des Thüringer Kreises (Eckartsberga, Freyburg, Sangerhausen, Weißensee, Sachsenburg, Langensalza) mit insgesamt 432.400 Einwohnern zufallen. Als Begrün- dung hieß es: Eine direkte Grenze zwischen Österreich und Preußen sei zu verhindern, weil sie immer wieder zu Reibungen zwischen beiden Mächten führen würde.

Der Wiener Kongress, der aus einem „System von Einzelberatungen "7 bestand, befasste sich zunächst mit anderen Fragen, unter anderem mit dem Schicksal Polens. Preußen, Österreich und England waren keineswegs bereit, Zar Alexander I. das Großherzogtum Warschau allein $\mathrm{zu}$ überlassen. Die „polnische Frage“ war eng mit der „sächischen Frage“ verknüpft, weil der König von Preußen bei Zugeständnissen an den Zaren von diesem erwartete, er werde eine vollständige Angliederung Sachsens an Preußen befürworten. Dass die beiden Verbündeten sich untereinander geeinigt hatten, beweist die Tatsache, dass die Leitung des Generalgouvernements des Königreichs Sachsen am 10. November 1814 dem preußischen Staatsminister Eberhard Freiherr von der Recke (1744-1816) sowie dem preußischen Generalmajor Friedrich Wilhelm Leopold Freiherr von Gaudi (1765-1832) übertragen wurde. Fürst Repnin erklärte den sächsischen Landesbehörden, er sei angewiesen, das Gouvernement den preußischen Bevollmächtigten zu übergeben, „um dadurch die Verbindung Sachsens mit Preußen, welche nächstens auf eine noch förmlichere Weise bekannt gemacht werden wird, einzuleiten, und beide Völker gleichsam zu verbinden". ${ }^{8}$ König Friedrich Wilhelm III. habe erklärt, Sachsen nicht als eine Provinz seiner Monarchie einzuverleiben, sondern es als eigenständiges Königreich unter Beibehaltung der Verfassung erhalten zu wollen.

Dieser eilige Vorstoß Russlands und Preußens, der nicht mit den anderen Mächten abgesprochen war, schuf zwar erst einmal Fakten, doch dürfte dies zu Verstimmungen bei den anderen Kongressteilnehmern geführt haben, und insbesondere Österreich gedachte nun eine vollständige Angliederung Sachsens an Preußen, die durchaus erwogen worden war, abzulehnen, weil die österreichische Seite ein zu starkes Vordringen Preußens und Russlands in den mitteleuropäischen Raum befürchtete. Das Einvernehmen zwischen Preußen und Österreich zerbrach.

Der Streit um die „sächsische Frage“ verdichtete sich im Dezember 1814. Metternich lehnte in einer Note an Hardenberg vom 10. Dezember 1814 eine Abtretung ganz Sachsens an Preußen ab. ${ }^{9}$ Um Preußen entgegenzukommen, schlug er eine Teilung des Lan-

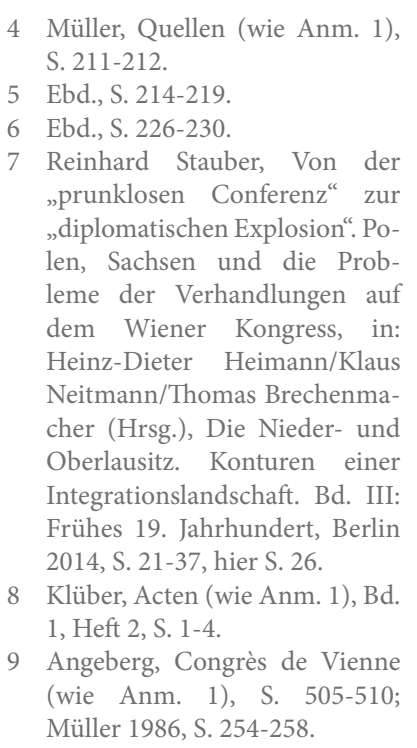




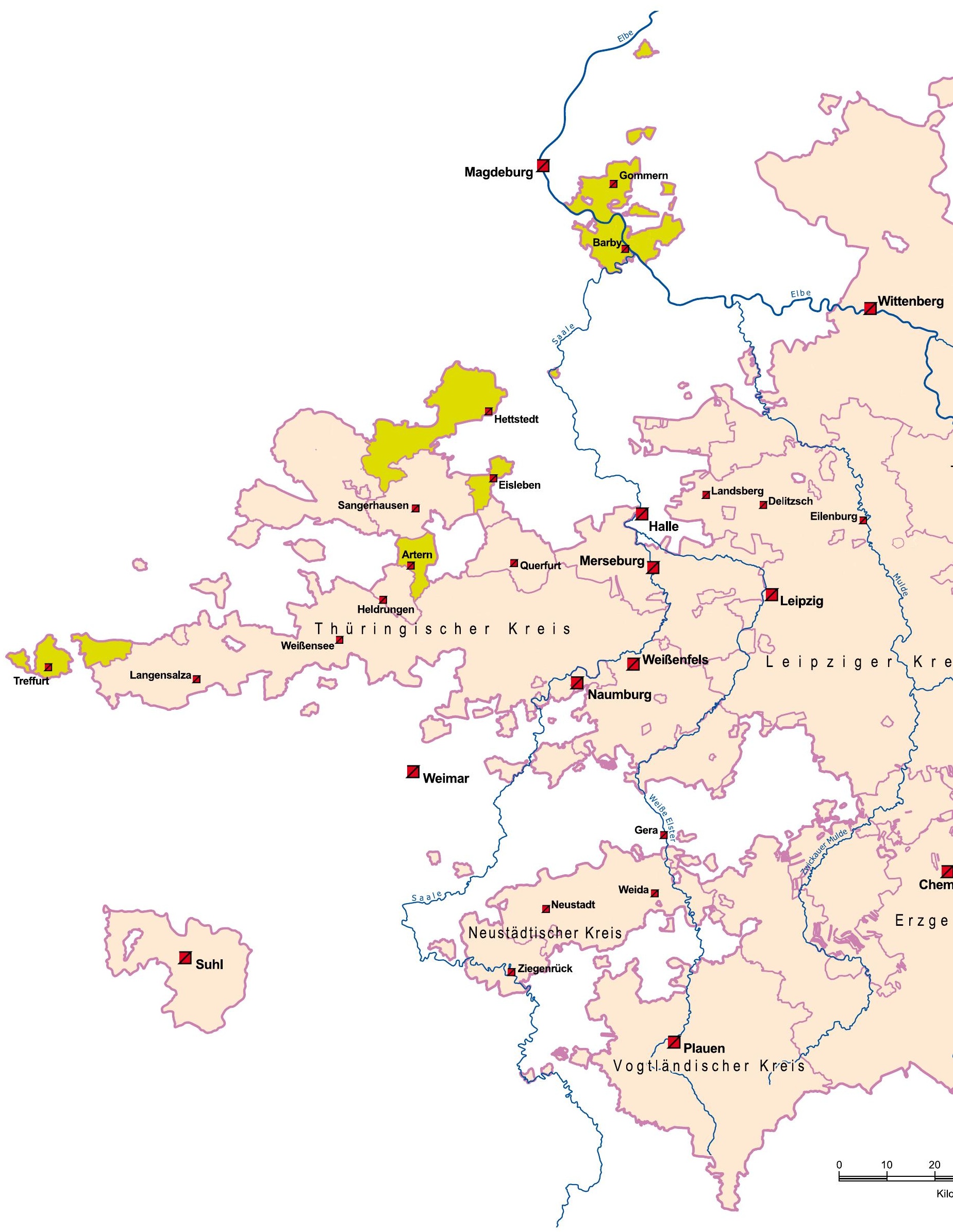




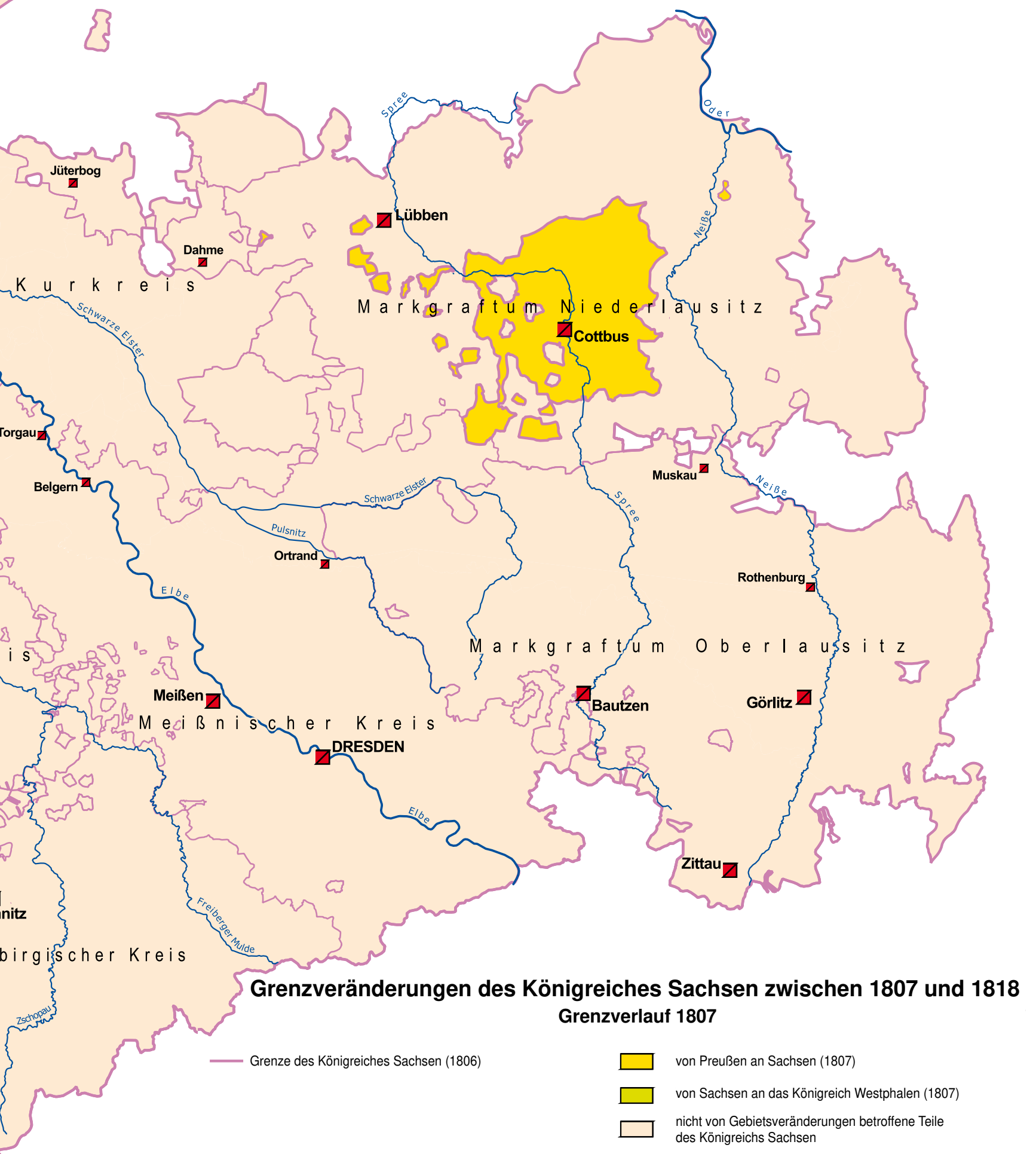

Kartographie: Uwe Ulrich Jäschke \& Rico llés Hochschule für Technik und Wirtschaft Dresden Auftraggeber: Sächsisches Staatsarchiv

Copyright

列

dem Sächsischen Staatsarchiv, Archivstraße 14, 01097 Dresden, vorbehalten. 
des vor. Der Note war ein Anhang beigefügt, in dem Preußen größere Gebietsgewinne zugesagt wurden. Der König von Preußen sollte Teile Polens, verschiedene Gebiete im Westfälischen Reichskreis sowie den nördlichen Teil Sachsens mit 432.400 Einwohnern erhalten. Zur Abtretung wurden jene Landesteile vorgeschlagen, die Wessenberg in seinem Papier vom Oktober 1814 aufgelistet hatte. Preußen wurde damit ein Fünftel des sächsischen Staatsgebiets angeboten.

Metternich wollte die vollständige Einverleibung Sachsens verhindern, um nicht auch noch am Erzgebirgskamm eine gemeinsame Grenze zwischen Preußen und Österreich zu erhalten, nachdem das preußische Schlesien schon an Böhmen und den österreichischen Teil Schlesiens grenzte. Da man auch nach einem Friedensschluss eine militärische Bedrohung durch Preußen erwartete, sollte ein „Pufferstaat“ nördlich des Erzgebirgskamms, möglichst unter Einschluss der Festung Torgau, die beiden Großmächte voneinander trennen. Metternich wollte sich ein militärisches Vorfeld erhalten, um allzu schnelle Angriffe Preußens auf Böhmen zu verhindern. Außerdem widersprach es seiner Vorstellung eines von Gott eingesetzten Königtums, einen legitimen König abzusetzen, zumal dieser König aus einer befreundeten (katholischen) und mit dem österreichischen Kaiserhaus verwandten Dynastie stammte.

Einen zu starken Machtzuwachs Preußens befürchteten nunmehr auch England und Frankreich. Am 19. Dezember 1814 übergab der französische Außenminister CharlesMaurice Herzog von Talleyrand-Périgord (1754-1838), der als Vertreter des besiegten Frankreich zunächst nicht an den Verhandlungen über Gebietsfragen zugelassen war, einen Brief an Metternich. Darin betonte er, dass mit einer "Aggressionsmacht“ („force d'aggression“) an der Grenze zu Böhmen kein Gleichgewicht der Mächte erreicht werden könne. Großbritannien wiederum wollte ein $\mathrm{zu}$ starkes Vordringen Russlands nach Mitteleuropa sowie ein zu starkes Preußen verhindern. Daher lehnte Castlereagh nunmehr ebenfalls eine vollständige Angliederung Sachsens an Preußen ab. Er bot sich an, in der „sächsischen Frage“ als Vermittler aufzutreten, wobei er offen für eine Teilung Sachsens warb. In Unterredungen am 19. und 20. Dezember 1814 mit Hardenberg und Fürst Adam Georg Czartoryski (1770-1861), der auf russischer Seite an den Verhandlungen teilnahm, versuchte er, Preußen und
Russland von einer vollständigen Annexion Sachsens abzubringen. ${ }^{10}$ Als realistischen Zugewinn für Preußen betrachtete er einen beträchtlichen Teil Nordsachsens mit den Festungen Wittenberg und Torgau sowie die Ober- und Niederlausitz. Dagegen beharrte Hardenberg unverändert auf dem preußischen Anspruch auf Sachsen und auf einer Entschädigung des Königs in Westphalen oder auf dem linken Rheinufer. ${ }^{11}$ Preußen werde eher in den Krieg ziehen, als nur einen Teil Sachsens zu akzeptieren. Der Kongress drohte somit an der „sächsischen Frage“ zu scheitern.

Um die verhärteten Fronten aufzubrechen, griff Castlereagh zu einem Verfahrenstrick: Da Preußen bei seinen Forderungen immer argumentierte, einen Ausgleich für die abzutretenden polnischen Gebiete erlangen $\mathrm{zu}$ wollen, schlug er vor, eine Kommission einzusetzen, die für jene zur Disposition stehenden Gebiete die Bevölkerungszahlen ermitten sollte. ${ }^{12}$ Die Entschädigungen sollten dabei nicht nach der Fläche, sondern nach der Zahl der Einwohner bestimmt werden. Castlereagh hoffte, bei einer genauen Berechnung der Einwohnerzahlen einen präzisen Plan für einen Bevölkerungsausgleich vorlegen $\mathrm{zu}$ können, der es Preußen ermögliche, einen Teilverzicht auf Sachsen zu leisten, denn letztlich standen fiskalische Interessen im Raum. Da die anderen Mächte den Vorschlag unterstützten, wurde innerhalb von nur vier Tagen eine mit Vertretern Österreichs, Preußens, Russlands, Frankreichs und Großbritanniens besetzte „Statistische Grundlagenkommission der Verbündeten Mächte“ („Commission chargée et poser des bases statistiques pour servir aux travaux des puissances réunies à Viennes") eingerichtet. Am Heiligabend $1814 \mathrm{kam}$ sie $\mathrm{zu}$ ihrer ersten Arbeitssitzung zusammen.

Am 27. Dezember 1814 beantragte der russische Gesandte Andrej Kyrillowitsch Rasumowsky (1752-1836) die Einberufung einer Konferenz der vier Mächte Russland, Österreich, Preußen und Großbritannien zur endgültigen Lösung der sächsischen Frage. Die erste dieser Konferenzen fand bereits am 29. Dezember statt. Österreich forderte, die „sächsische Frage“ unter Beteiligung und Zustimmung des Königs von Sachsen zu lösen, was Preußen und Russland aber kategorisch ablehnten. Auch die zweite Konferenz am 31. Dezember brachte keinen Fortschritt. Die Interessengegensätze waren so groß, dass der Ausbruch eines Krieges zwischen den Mächten möglich schien. Harden- 
berg drohte, würden Österreich und Großbritannien ihre Zustimmung zur Angliederung Sachsens verweigern, so betrachteten das Preußen und Russland als Kriegserklärung. Unter dem Eindruck dieser Drohungen schlossen Österreich, Großbritannien und Frankreich am 3. Januar 1815 ein gegen Russland und Preußen gerichtetes Bündnis, in dem sie sich bei einem Angriff gegenseitige militärische Hilfe zusicherten. ${ }^{13}$ Die unverhohlene Drohgebärde führte schließlich $\mathrm{zu}$ einem Einlenken Preußens in der „sächsischen Frage“. Zwar gab Hardenberg vor, nach wie vor auf der vollständigen Eingliederung Sachsens $\mathrm{zu}$ bestehen. Der König von Sachsen solle mit einem Gebiet am linken Rheinufer abgefunden werden. Doch unter dem Druck der anderen Mächte war er dann doch $\mathrm{zu}$ einer Kompromisslösung bereit.

Zur Sitzung am 12. Januar 1815, zu der erstmals Frankreich zugelassen war, legte Metternich einen Gegenvorschlag („Contre-projet austrichien“) vor, in dem er auf den Erhalt eines Kerns des Königreichs Sachsen beharrte, aber dem preußischen König größere Zugeständnisse machte. ${ }^{14}$ Die Grenzlinie sollte in der Oberlausitz zunächst der Wittig und dann der Neiße folgen, so dass Görlitz auf sächsischer Seite geblieben wäre. Dann sollte eine Linie südlich von Rothenburg, das an Preußen gefallen wäre, über Königswartha und Wittichenau bis zur Schwarzen Elster und weiter entlang dieses Flusses bis zur Elbe gezogen werden. Zwischen Belgern und Torgau sollte die Elbe die Grenze bilden. Damit wäre die Festung Torgau noch auf sächsischer Seite geblieben. Nördlich von Leipzig sollte die Grenzen entlang der Linie Torgau Eilenburg - Delitzsch - Landsberg verlaufen. Westlich von Leipzig war die Saale als Grenzlinie vorgesehen, und zwar so, dass Zeitz, Weißenfels und Naumburg sächsisch geblieben wären, während Merseburg auf preußischer Seite gelegen hätte. Dem König von Preußen wurde somit eine Hälfte der Oberlausitz, die Niederlausitz, der Wittenberger Kreis, Teile des Leipziger Kreises, der überwiegenden Teil des Thüringer Kreises, der gesamte Neustädter Kreis sowie die sächsischen Anteile der Grafschaften Henneberg und Mansfeld angeboten. Insgesamt wären 782.249 von 2.085.911 Einwohnern unter preußische Hoheit gelangt.

Bei der Grenzziehung fällt auf, dass Metternich nicht Castlereaghs Vorschlag folgte, beide Lausitzen an Preußen abzutreten. Das hätte bedeutet, dass das Königreich Preußen im Bereich der Oberlausitz bis an die böhmische Grenze vorgerückt wäre. Um das zu verhindern, schlug Metternich eine Teilungslinie durch die Oberlausitz vor. Außerdem versuchte er, die Festung Torgau und die größeren Städte an der Saale für Sachsen zu sichern. Die Militärs am österreichischen Hof hatten darauf gedrängt, dass die Festungen Erfurt und Torgau keinesfalls in preußische Hände fallen dürften. Auch Bautzen und Zittau müssten in sächsischer Hand bleiben.

Am 19. Januar 1815 veröffentlichte die statistische Kommission ein genaues Verzeichnis der Einwohnerzahlen der Kreise und Ämter des Königreichs Sachsen. ${ }^{15}$ Am 28. Januar berieten die Mächte nochmals Metternichs Teilungsplan. Dabei wurden die Einwohnerzahlen nach den Angaben der Statistischen Kommission nachgerechnet. Dabei kam man zu dem Ergebnis, dass in dem für Preußen vorgesehenen Gebiet nur 723.311 und nicht 782.249 Einwohner lebten. Unter Berücksichtigung auch der anderen Territorien in Nordund Westdeutschland, die Metternich in seinem "Contre-projet" Preußen zugestehen wollte, ergab die Nachrechnung ein Defizit von 264.311 Einwohner, die folglich durch zusätzliche Gebietsabtretungen an Preußen gelangen sollten. ${ }^{16}$

Nachdem Hardenberg schon am 13. Januar angedeutet hatte, dem Erhalt eines stark verkleinerten Königreichs Sachsen mit der Umgebung Dresdens und Teilen des Erzgebirges zustimmen zu können, wurden die Verhandlungen am 28. Januar 1815 fortgesetzt. Dabei erreichte Metternich die grundsätzliche Zustimmung der preußischen Seite zu einer Teilung Sachsens. Die erst unter dem 8. Februar in den Kongressakten veröffentlichten Aufzeichnungen Hardenbergs lassen erkennen, dass sich Preußen allerdings nicht mit der von Österreich vorgeschlagenen Teilungslinie zufrieden gab. ${ }^{17}$ Hardenberg bemängelte, dass nach Metternichs Plan von den 28 größten Städten des Königreich Sachsens nur acht zu Preußen kommen sollten. Das sei nicht hinnehmbar. Außerdem forderte er Leipzig für Preußen. Ohne den Gewinn einer der beiden bedeutendsten Städte Sachsens, Dresden oder Leipzig, könne es keine Einigung geben.

Unterdessen verhandelte Castlereagh weiter. Er versuchte, die preußische Seite zum Verzicht auf Leipzig zu bewegen, indem er andere territoriale Zugeständnisse anbot. Am 29. Januar notierte Castlereagh, dass Österreich bereit sei, die Festungen Torgau und Erfurt
13 Angeberg, Congrès de Vienne (wie Anm. 2), S. 589-592.

14 Ebd., S. 604, 680-683.

15 Ebd., S. 648-651.

16 Ebd. S. 722.

17 Ebd., S. 707-712. 


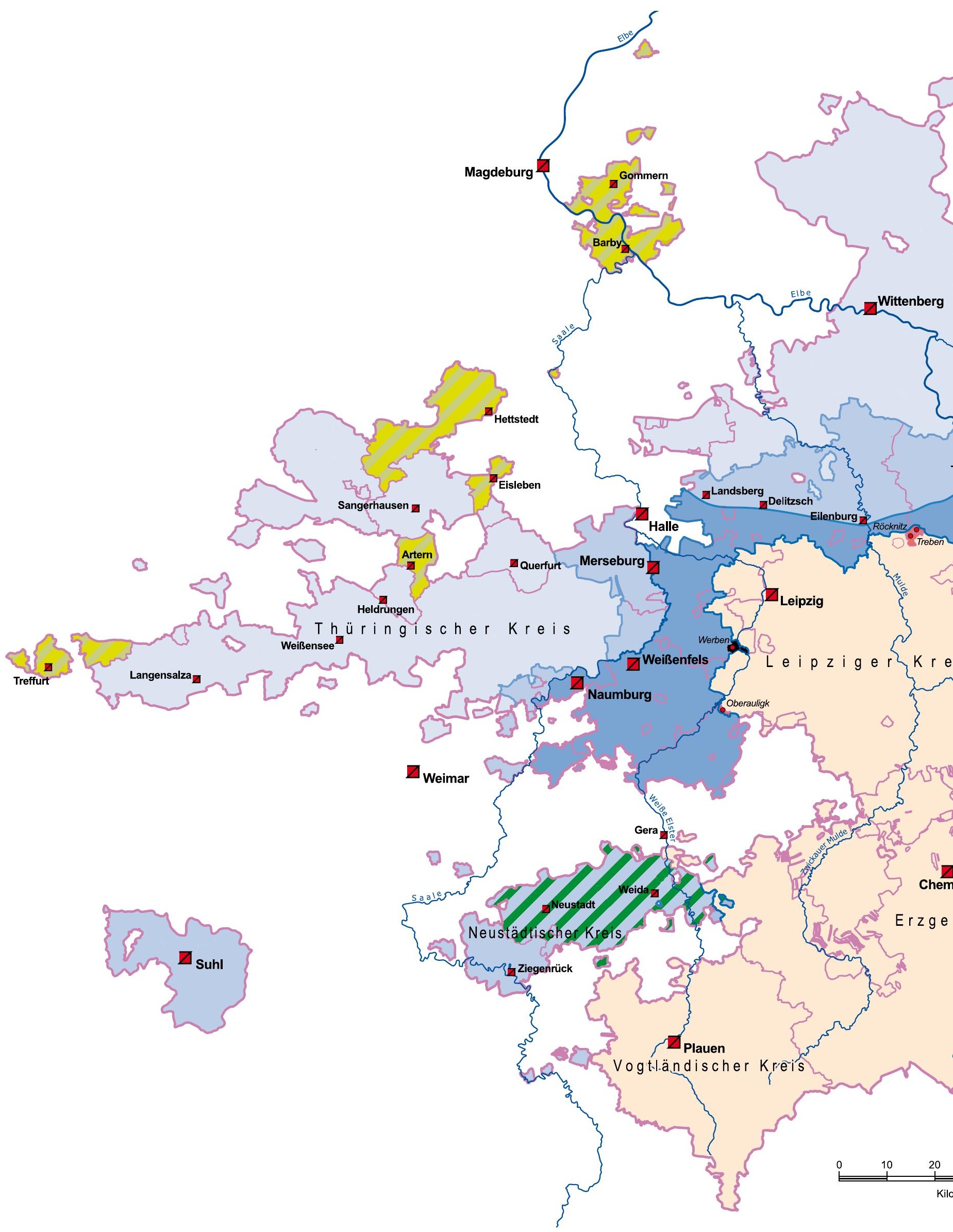




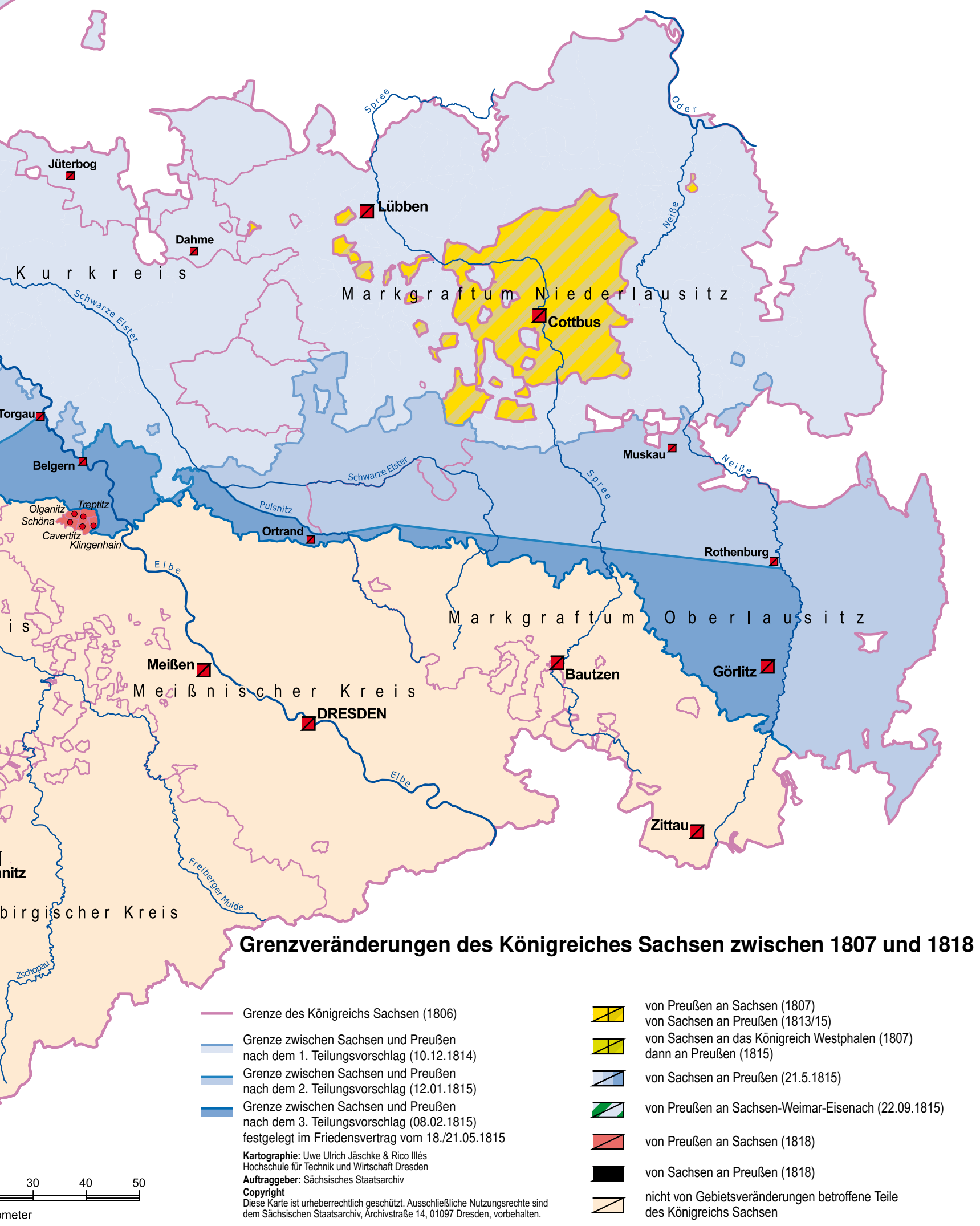




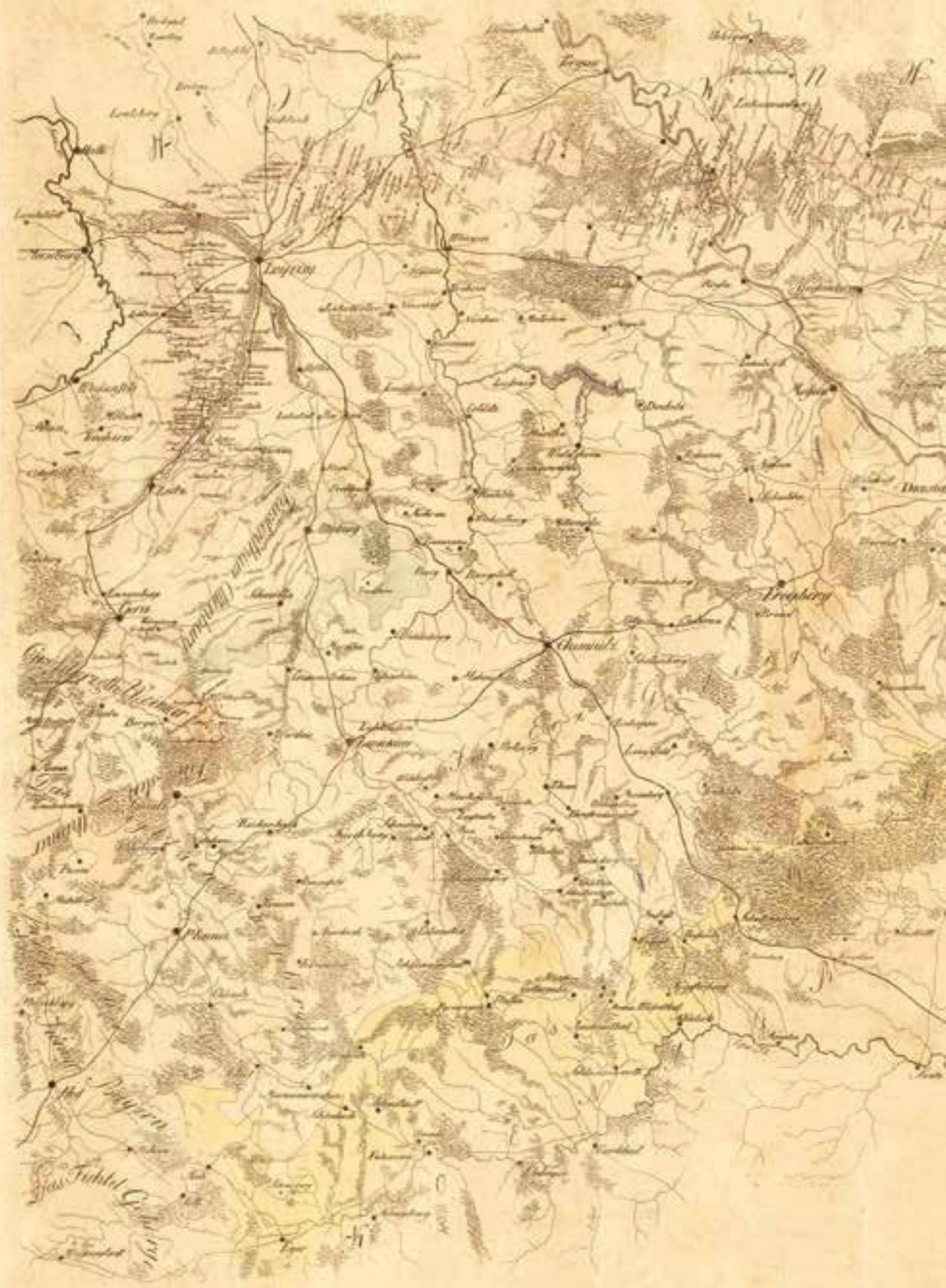




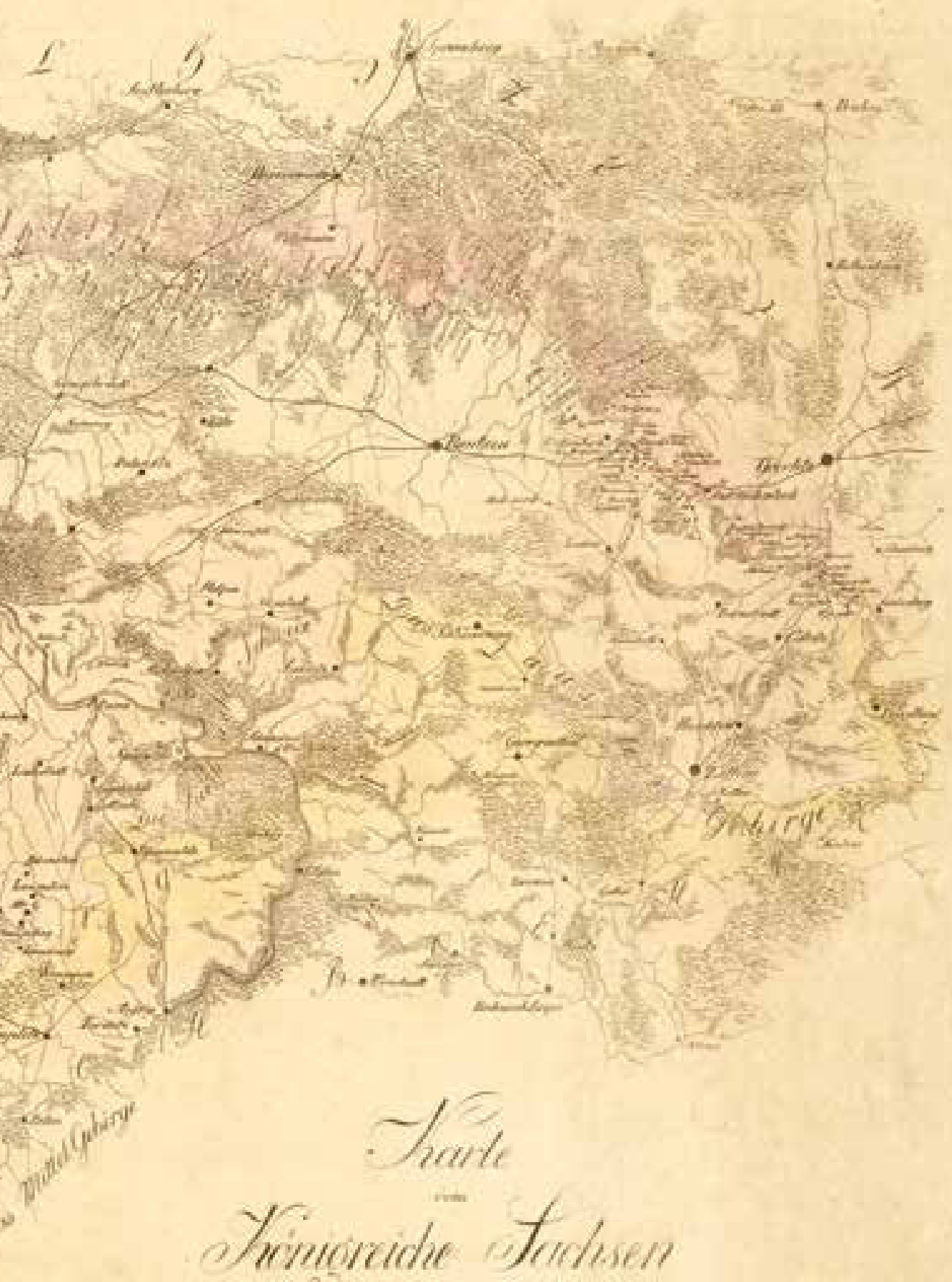

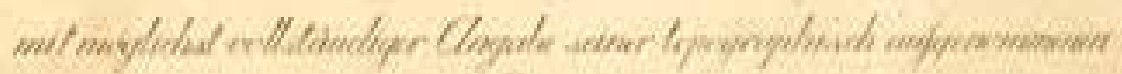
Gremier!

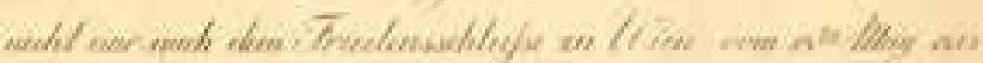

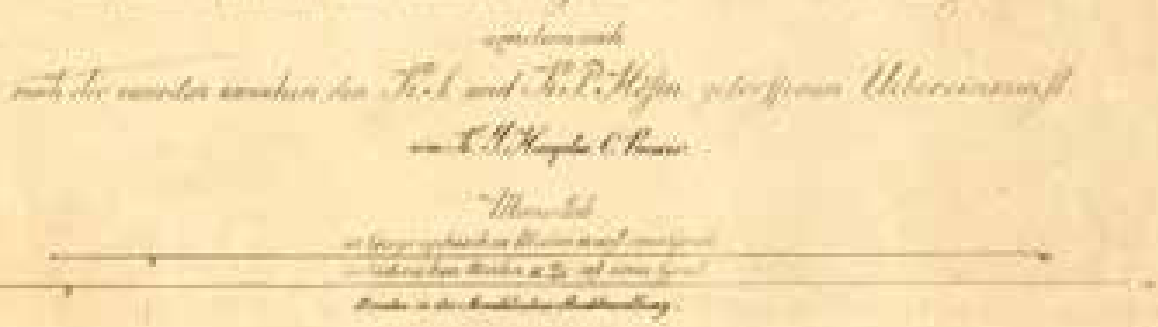

Karte des Königreichs Sachsen mit Eintragung der 1815 


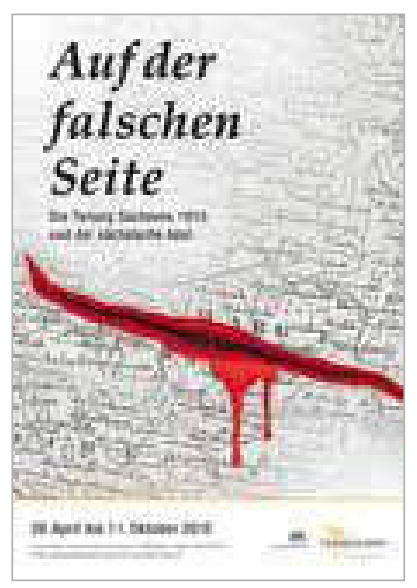

Der Landesteilung des Jahres 1815 widmet sich die Sonderausstellung "Auf der falschen Seite" im Schloss Nossen. Die von Dr. Lars-Arne Dannenberg und Dr. Matthias Donath kuratierte Ausstellung ist bis zum 11. Oktober 2015 geöffnet.

\section{Schloss Nossen}

Am Schloss 3

01683 Nossen

Tel.: 035242-50435

nossen@schloesserland-sachsen.de

www.schloss-nossen.de

Öffnungszeiten:

$\mathrm{Di}-\mathrm{Fr} \quad 10.00-17.00 \mathrm{Uhr}$

Sa/So $10.00-18.00 \mathrm{Uhr}$ den Preußen zu überlassen, wenn diese auf Leipzig verzichteten. ${ }^{18}$ In den folgenden Tagen wurde offenbar hartnäckig um Leipzig wie auch um größere Gebietsabtretungen in der Oberlausitz gerungen. Castlereagh hatte am 29. Januar ein Gespräch mit Hardenberg, in dem es um „Leipsick and the re-entering angle into Bohemia by Bautzen and Zittau“ ging. ${ }^{19}$ Aus der Formulierung scheint hervor, Hardenberg habe die südliche Oberlausitz und damit eine direkte Grenze zu Böhmen gefordert. Am 30. Januar sprach Castlereagh mit König Friedrich Wilhelm III. von Preußen, der jedoch einen Verzicht auf Leipzig ablehnte. ${ }^{20}$ Einen Ausweg aus der verfahrenen Situation ebnete Zar Alexander I., der im Gespräch mit Castlereagh zusagte, den Preußen die Stadt Thorn an der Weichsel zu überlassen, die ursprünglich wie Krakau ein neutrales Gebiet werden sollte. Thorn war für die militärische Sicherheit Preußens ungleich wichtiger als Leipzig, so dass der preußische König dann doch Anfang Februar für Thorn auf das bevölkerungsstärkere Leipzig verzichtete. ${ }^{21}$ Castlereagh berichtete am 6. Februar, dass nach der russischen Abtretung Thorns an Preußen die letzten Schwierigkeiten in der „sächsischen Frage“ überwunden seien. ${ }^{22}$

Der Durchbruch in der „sächsischen Frage“ gelang am 8. Februar 1815. Die fünf Großmächte einigten sich auf einen modifizierten Teilungsvorschlag. ${ }^{23}$ Er sah gegenüber Metternichs Vorschlag zusätzliche Gebietsabtretungen mit ingesamt 131.469 Einwohnern vor. Preußen erhielt das Gebiet um Görlitz zugeprochen, nicht aber die südliche Oberlausitz, außerdem größere Anteile der Ämter Großenhain, Torgau, Delitzsch, Weißenfels, Merseburg und Zeitz sowie das gesamte Amt Mühlberg. Hatte Metternich noch einen Grenzverlauf entlang von Elster, Elbe und Saale vorgesehen, so wurde die Grenzlinie nun so weit in das sächsische Kernland hineinverlegt, dass die Städte Görlitz, Elsterwerda, Mühlberg, Torgau, Eilenburg, Delitzsch, Weißenfels, Naumburg, Merseburg und Zeitz unter preußische Hoheit kamen. Den größten Zugewinn gegenüber dem Teilungsvorschlag vom 12. Januar erzielte Preußen an der Saale, wo die Stifter Naumburg und Merseburg sowie das Amt Weißenfels fast vollständig der preußischen Regierung unterstellt wurden, weiterhin an Mulde und Elbe sowie in der Oberlausitz. Insgesamt lebten im Abtretungsgebiet 855.305 Einwohner.

Die Teilung Sachsens wurde gegen den langen, energischen Widerstand der preußi- schen Seite beschlossen, die das ganze Königreich in Besitz nehmen wollten. Österreich und England konnten ein „Restsachsen" unter Herrschaft des sächsischen Königs nur retten, indem sie der Gegenseite eine Teilung vorschlugen. Die letztlich ausgehandelte Teilungslinie berücksichtigte das militärische Interesse Österreichs, auf sächsischem Gebiet keine gemeinsame Grenze mit Preußen zu erhalten, und das fiskalische Interesse Preußens, sich möglichst viele Einwohner und damit steuerpflichtige Untertanen zu sichern. Das Ringen um ein möglichst großes „Restsachsen“ nördlich des Erzgebirges unter Einschluss der südlichen Oberlausitz, eine Forderung Österreichs, bei gleichzeitiger Abtretung ausreichend großer Einwohnerzahlen an Preußen führte dazu, dass die Grenzen auf dem Konferenztisch mitten durch geschlossene Herrschafts- und Amtsbezirke gezogen wurden. Dass man dabei Grundherrschaften, Kirchgemeinden und andere zusammenhängende Einheiten zerschnitt, wussten die Diplomaten nicht, als sie - einzig anhand von Landkarten und Einwohnerzahlen - die Grenze festlegten.

Letzlich handelte es sich bei der Grenzziehung des Wiener Kongresses um einen Kompromiss. Preußen musste auf das sächsische Kernland mit Dresden und Leipzig verzichten, das es eigentlich haben wollte. Österreich hätte eine Grenzziehung entlang der historischen Grenzen der Kreise und Ämter wahrscheinlich durchsetzen können, hätte aber dann den Preußen wohl grenznahe Gebiete im Süden Sachsens überlassen müssen. Daher gab man das Prinzip des ersten Teilungsvorschlags, entlang historischer Grenzen zu teilen, auf. Aber auch das Prinzip des zweiten Teilungsvorschlags, die Grenzen möglichst an den Flüssen verlaufen $\mathrm{zu}$ lassen, konnte nicht umgesetzt werden, weil so nicht ausreichend Untertanen an Preußen abgetreten werden konnte. Da Metternich aus militärischen Gründen Preußen unbedingt von der Nordgrenze Böhmens fernhalten wollte, stimmte er lieber einer Zerteilung der Oberlausitz, des Meißner Kreises und des Leipziger Kreises zu. Bautzen und Zittau wurden aus strategischen Erwägungen auf der sächsischen Seite belassen und gegen Görlitz, Naumburg und Weißenfels eingetauscht.

Vertreter Sachsens saßen in Wien nicht mit am Verhandlungstisch. Allerdings war der sächsische König durch seinen Bevollmächtigten, Friedrich Albrecht Graf von der Schulenburg-Klosterrode (1772-1835), über 
den Fortgang der Verhandlungen informiert. Dieser durfte allerdings nur als „Privatmann" Gespräche führen. Nachdem sich die Großmächte auf den Erhalt eines „Restsachsen“ verständigt hatten, durfte Friedrich August I. im Februar 1815 das Schloss Friedrichsfelde bei Berlin verlassen. Auf Einladung des österreichischen Kaisers reiste er nach Preßburg (heute Bratislava, Slowakei). Der König wollte erst nicht einer Abtretung bedeutender Landesteile an Preußen zustimmen. Dann machte er die Abtretung von Bedingungen abhängig. Doch die Großmächte, die sich mühevoll auf den Teilungsplan verständigt hatten, zeigten keinerkei Bereitschaft, auf die Forderungen einzugehen. Da man dem sächsischen König drohte, er werde alles verlieren, stimmte er der Landesteilung schließlich zu.

Am 3. Mai 1815 begannen Vertragsverhandlungen zwischen Sachsen, Österreich, Preußen und Russland. Darin stimmte der König von Sachsen der bereits ausgehandelten Teilungslinie $\mathrm{zu}$, auf deren Verlauf er keinen Einfluss mehr hatte. Dass man den König von Sachsen nunmehr als Verhandlungspartner akzeptierte, lag an dem Legitimitätsprinzip des Wiener Kongresses. Es sollte der Anschein aufrecht erhalten werden, der König entscheide als Souverän. Faktisch hatte er aber nur noch einem Diktat der Großmächte zuzustimmen.

Der Friedensvertrag Sachsens mit Preußen und Russland, der die Gebietsabtretungen regelte, wurde am 18. Mai 1815 in Preßburg ausgefertigt. Nach der Ratifizierung durch den sächsischen König trat er am 21. Mai in Kraft. Die wichtigsten Regelungen wurden auch in die Schlussakte des Wiener Kongresses übernommen, die die Signatarmächte zwischen dem 6. und dem 26. Juni 1815 unterzeichneten.

König Friedrich Wilhelm III. von Preußen nahm bereits am 22. Mai 1815 von dem „mit der Preußischen Monarchie vereinigten Antheil von Sachsen" Besitz. ${ }^{24}$ Mit dieser Erklärung nahm er die Titel eines Herzogs von Sachsen, Markgrafen der beiden Lausitzen, Landgrafen von Thüringen und gefürsteten Grafen von Henneberg an. Die Verwaltung des Generalgouvernements wurde von Dresden nach Merseburg verlegt. Dort errichtete man das "Generalgouvernement des Herzogthums Sachsen“, das noch 1815 in kleinere Verwaltungseinheiten zerlegt wurde. Die Landesteile um Merseburg und Naumburg bildeten fortan den Regierungsbezirk Merseburg der neu geschaffenen Pro-

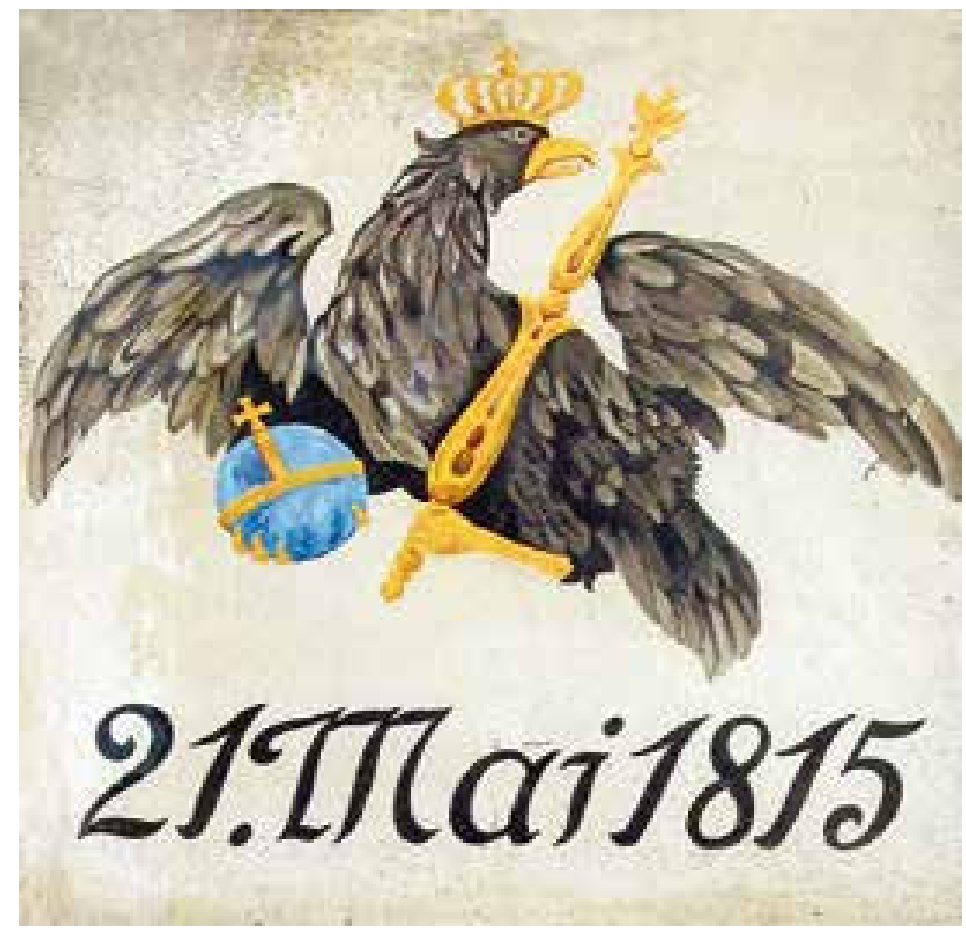

vinz Sachsen, die neben den preußisch gewordenen Teilen Thüringens und Sachsens mit der Altmark auch ein ehemals brandenburgisches Gebiet umfasste. Die nördlichen Landesteile Sachsens, der Wittenberger Kreis und die Niederlausitz, wurden in die Provinz Mark Brandenburg eingegliedert. Den preußischen Anteil des Markgraftums Oberlausitz schloss man dem Regierungsbezirk Liegnitz der Provinz Schlesien an. Damit waren die „Musspreußen“ nunmehr auf drei Provinzen aufgeteilt, was eine Besinnung auf die gemeinsame sächsische Vergangenheit verhinderte.

Der König von Preußen hatte sich in der Schlussakte des Wiener Kongresses verpflichtet, seinem Verbündeten, dem Großherzog Carl August von Sachsen-WeimarEisenach (1757-1828), an Weimar angrenzende Gebiete mit mindestens 50.000 Einwohnern abzutreten. Daher übergab er ihm mit Staatsvertrag vom 22. September 1815 einen Teil der "erbeuteten“ sächsischen Gebiete. So gelangten zwei Drittel des Neustädter Kreises mit den Städten Neustadt/ Orla, Triptis, Auma, Weida und Berga/Elster nur vier Monate nach der preußischen Besitzergreifung an Sachsen-Weimar. Carl August von Sachsen-Weimar-Eisenach hatte gehofft, selbst König von Sachsen und Thüringen zu werden oder zumindest Erfurt und Fulda zu erwerben. Er musste sich mit wenigen Gebietsgewinnen und dem Titel eines Großherzogs zufrieden geben.
Tafel an einem Stadttor in Delitzsch mit dem preußischen Adler und dem Tag der preußischen Inbesitznahme

18 Müller, Quellen (wie Anm. 1), S. 291-294.

19 Ebd., S. 295-297.

20 Ebd.

21 Thorn und Umland wurde in den Auflistungen der Statistischen Kommission mit 20.000 Einwohnern und Leipzig mit 30.796 Einwohnern bewertet.

22 Müller, Quellen (wie Anm. 1), S. 295-297.

23 Angeberg, Congrès de Vienne (wie Anm. 1), S. 722-724.

24 Patent wegen der Besitzergreifung des mit der Preußischen Monarchie vereinigten Antheils von Sachsen, 22. Mai 1815 